\title{
An Iterative Graph-based Image Restoration using Data-Adaptive Objective Function
}

\author{
P. Hema Sree \\ Assoc. Professor, CVR College of Engineering/ ECE Department, Hyderabad, India. \\ Email: phemasree1@yahoo.co.in
}

\begin{abstract}
An image is defined as a function of weighted graph encoded with Laplacian matrices and its associated kernel similarities. An Iterative Graph (IG)-based Image Restoration with data-adaptive objective function is used to deblur the images that are degraded due to unconstrained conditions. From a normalized graph Laplacian, cost function is defined with a new regularization term and new data fidelity term. From the fast symmetry preserving balancing matrix, the normalizing coefficients are derived. This results in determining the spectral properties like symmetric, positive semi-definite and returning zero vector when applied to a constant Image. This algorithm has inner and outer iterations. In the inner conjugate gradient iterations, an updated objective function is minimized and the similarity weights are recalculated with earlier estimate in each outer iterations. The performance of this method is more effective for various restoration problems like sharpening, deblurring and denoising. Experimental results show that IGbased algorithm performs more powerfully in terms of objective criteria and visual quality.
\end{abstract}

Index Terms: Balancing matrix, Graph Laplacian, Conjugate Gradient, Deblurring, Kernel similarity matrix, Normalizing coefficients.

\section{INTRODUCTION}

Many of the real world pictures or images are degraded in some sense due to variation in environmental conditions, camera setting, relative motion between camera and subject, etc. The purpose of restoration algorithm is to undo the undesirable distortions like noise, blur from a degraded image. This paper concentrates mainly on the distortions caused by blurring. The blurring process in linear shift invariant Point Spread Function (PSFs) is represented by a linear model as

$$
y=A f+n
$$

where, $y$ is $\mathrm{N} x \mathrm{~N}$ ordered vector representation of a input blurred and noisy image, latent image in vector form is represented as $f, n$ is the noise vector which is independent and equivalently distributed zero mean noise with standard deviation $\sigma$. Based on PSFs and type of assumptions, Blurring matrix $A$ of size $\mathrm{N}^{2} \times \mathrm{N}^{2}$ is constructed.

Frequently used deblurring methods depend on optimizing the cost function which is expressed in the form

$$
E(f)=\|y-A f\|^{2}+\eta R(f)
$$

with respect to unknown image vector $f$. The first term in above equation is 'data fidelity term' and the second term is 'prior term' which is used for regularizing the ill-posed problem. The amount of regularization is controlled by parameter $\eta$. Based on nature of blurs and type of regularization parameter, many deblurring algorithms are classified. Most of the deblurring algorithms are based on Total Variation (TV)-type regularization [1]. Specific definition of TV term is varied and suitable optimization methods are chosen to define the cost function. Nonlocal differential operator as regularization term with different norms is used in other methods [2]. Example-based manifold priors are used for regularization in [3]. The prior term is added to estimate a gradient distribution in [4]. Hessian norm regularization is used to solve deblurring problem in biomedical applications [5]. BM3D method is one of the most recent algorithm uses generalized Nash equilibrium approach [6], which balances the objective functions for denoising and deblurring. This algorithm is best deblurring method for symmetric blurs like Out-of-focus and Gaussian blurs. A hyper-Laplacian [7], is based on statistics is used for motion deblurring applications. In [8] a progressive inter-scale, intrascale approach is used for non-blind deconvolution. Different derivative terms are used as cost function for motion deblurring [9]. The proposed approach is kernel similaritybased image deblurring with novel data adaptive objective function. This approach can also be used for image denoising and sharpening.

The block diagram of the proposed iterative deblurring method is shown in Fig. 1. This method consists of outer and inner iterations. In each outer iteration $\mathrm{k}$, an updated objective function is minimized and corresponding estimate $\mathrm{f}^{\prime}(\mathrm{k})$ is obtained in inner iterations using Conjugate Gradient (CG).

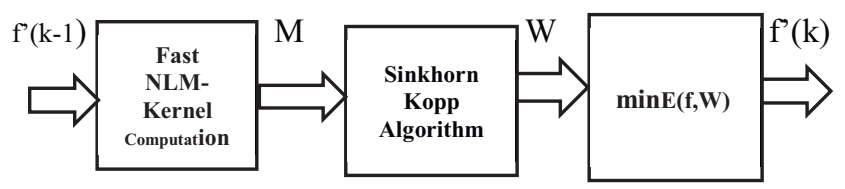

Figure 1. Block diagram of Iterative Graph (IG)-based Image Restoration method

The cost function used in the proposed method is based on new interpretation of a normalized graph Laplacian. It contains a normalized regularization term and data fidelity term. Fast symmetry preserving matrix balancing algorithm is used to extract the Normalizing coefficients [10], which results in spectral properties (symmetric, positive semidefinite) for the graph Laplacian. As the cost function is considered in quadratic form, the filtering operation of the objective function represents the spectral analysis for the restoration algorithm. This results in effective implementation of the algorithm using Conjugate Gradient 
(CG) and fast sparse matrix-vector products. Kernel similarity values are initially computed differently by considering blurry image and denoised form of the noisy input. The contribution of ringing and noise articrafts is avoided. Inner CG iterations are stopped when the denoised image is exploited as plug-in estimator of blurred clean image $A f$. This is used in Prediction of Mean Squared Error (PMSE) measure. Performance of this algorithm is improved by updating the weights during the outer iterations. These are computed from similarity weights of the input image through outer iterations from their enhanced versions. Denoising and Sharpening methods are used as a special case in this proposed cost function. This approach can handle different varieties of Motion and symmetric blur PSFs.

The paper is organized as follows: Section II discusses on how to derive filtering and symmetric kernel similarity matrices, which are basic building blocks of the algorithm. Further the spectral properties of normalized Laplacian matrix are discussed. Section III presents the objective function and proposed procedure to optimize it for final estimate. Section IV focuses on the implementation details. Experimental evaluation for Gaussian, motion and out-offocus PSFs are presented in section V. Section VI is devoted to conclusion and discussion on future directions.

\section{DeRivation OF BASIC BUILding BLOCK MATRICES}

Kernel similarity matrix $\mathrm{M}$ and doubly stochastic symmetric matrix $\mathrm{W}$ are the main building blocks in the algorithm. A normalized Laplacian matrix is defined from these matrices whose spectral properties are significant for analyzing the nature of the algorithm.

A. Kernel Similarity Matrix (M) and Filtering Matrix (W)

From the valid kernel similarity function in [11], each $(i, j)$ th element of the kernel similarity matrix $\mathrm{M}$ is calculated using non-local means (NLM) as [12]

$$
M(i, j)=\exp \left(-\frac{\left\|\left({f^{\prime}}_{i}-f^{\prime}{ }_{j}\right)\right\|^{2}}{k^{2}}\right)
$$

where $\mathrm{f}{ }_{\mathrm{i}}$ and $\mathrm{f}{ }_{\mathrm{j}}$ are strip patches around the pixels $\mathrm{i}$ and $\mathrm{j}$ of a image $f^{\prime}$ and $k$ is called smoothing parameter. From the estimate of previous iteration, kernel similarity weights (M) in each outer iteration are recomputed. The resultant $M$ is a symmetric non-negative matrix. $M$ matrix is sparse, as it computes the similarity between each patch and neighborhood patches around it (window size is $9 \times 9$ ). The Sinkhorn matrix balancing procedure [13] is applied to matrix $\mathrm{M}$ that results in doubly stochastic filtering matrix $\mathrm{W}$, where $\mathrm{W}=\mathrm{L}^{-1 / 2} \mathrm{ML}^{-1 / 2}(\mathrm{~L}=$ diagonal scaling matrix. As $\mathrm{W}$ is symmetric, it can be decomposed with orthonormal matrix $\mathrm{O}$ as $\mathrm{W}=\mathrm{OSO}^{\mathrm{T}}$. The columns of $\mathrm{O}$ are Eigen vectors of $\mathrm{W}$ and $\mathrm{S}$ is a diagonal matrix that consists of Eigen values of $\mathrm{W}$ as its diagonal matrix, $S=\operatorname{diag}\left\{\lambda_{1}, \lambda_{2}, \lambda_{3}, \ldots . \lambda_{N}\right\}$. Applying $W$ to a signal, preserves the DC component of the signal, as the largest Eigen value is equal to 1 exactly with corresponding DC vector. This property is more important in filtering operations and the spectral analysis of $\mathrm{W}$ matrix reveals its explicit low pass nature.

\section{B. Normalized Graph Laplacian matrix}

The normalized graph Laplacian matrix for image filters is defined as

$$
I-W=I-L^{\frac{-1}{2}}
$$

The set of Eigen vectors of I-W are considered as basis function of the graph and its Eigen values are treated as graph frequencies. Basically the Laplacian of I-W is high pass in nature and it can be interpreted as data adaptive Laplacian filter when applied to an image [14]. Based on the type of application, it integrates different filters in data term which is attached with regularized term. From the concept of Marcov chains, element $(i, j)$ of $\mathrm{D}^{-1} \mathrm{M}$ represents the probability of shifting from one node $i$ to node $j$ of the graph in one step [15]. In Image deblurring applications, they provide fast methods in solving large linear system of equations when optimizing with $\mathrm{CG}$ methods. The linear equations are symmetric and positive definite. This normalized graph Laplacian I-W provides better performance than with unnormalized graph Laplacian.

The difference operator to the proposed normalized graph Laplacian is represented as

$$
d f(i, j)=\sqrt{M(i, j)}\left(\frac{f(j)}{\sqrt{L(j, j)}}-\frac{f(i)}{\sqrt{L(i, i)}}\right)
$$

where $\mathrm{L}(\mathrm{i}, \mathrm{i})$ and $\mathrm{L}(\mathrm{j}, \mathrm{j})$ corresponds to ith and $\mathrm{jth}$ elements of the diagonal matrix L, derived from Sinkhorn matrix balancing algorithm [13]. Considering the divergence operator [16] with Laplace operator, the normalized Laplacian $\mathrm{I}_{-} \mathrm{L}^{-1 / 2} \mathrm{ML}^{-1 / 2}$ is represented as

$$
\Delta f(i)=\frac{1}{\sqrt{L(i, i)}} \sum_{j, j \sim i} M(i, j)\left(\frac{f(i)}{\sqrt{L(i, i)}}-\frac{f(j)}{\sqrt{L(j, j)}}\right.
$$

Thus the resultant regularization term is obtained a

$$
\begin{aligned}
& R(f)=\frac{1}{2} \sum_{i=0}^{N} \sum_{j=i} M(i, j)\left(\frac{f(i)}{\sqrt{L(i, i)}}-\frac{f(j)}{\sqrt{L(j, j)}}\right)^{2} \\
& R(f)=f^{T}(I-W) f
\end{aligned}
$$

The Laplace operator in equation (6) is a second order derivative operator, which represents the effect of normalized laplacian at each pixel $i$, when applied to an input vector $\mathbf{f}$.

\section{DEBLURRING MeTHOD}

This algorithm consists of inner and outer iterations. Data adaptive matrix $\mathrm{M}$ is computed to estimate the unknown image initially and it is gradually improved though iterations. The matrix $\mathrm{W}$ is estimated in each iteration and used to minimize the given objective with unknown image $\mathbf{f}$

$$
E(f)=(y-A f)^{T}(I+\beta(I-W))(y-A f)+\eta z^{T}(I-W) f
$$

where $\beta>-1$ and $\eta>0$ are parameters related to noise and blur. First term determines the blurred and filtered version of Input $\mathbf{y}$. Frequency selectivity is defined by $\beta$, based on amount of blur and noise. Second term is adaptive-data difference term which is based on normalized Laplacian matrix I-W. The filtering process of the cost function in [17] is more influenced on the objective function as

$$
E(f)=\left\|\{I+\beta(I-W)\}^{1 / 2}(y-A f)\right\|+\eta\left\|(I-W)^{1 / 2} f\right\|
$$

the term $\mathrm{I}+\beta(\mathrm{I}-\mathrm{W})=\mathrm{O} \Lambda \mathrm{O}^{\mathrm{T}}$ is semi-definite and symmetric matrix. The matrix $\{\mathrm{I}+\beta(\mathrm{I}-\mathrm{W})\}^{1 / 2}=\mathrm{O} \Lambda^{1 / 2} \mathrm{O}^{\mathrm{T}}$ has filtering 
nature identical to that of $\mathrm{I}+\beta(\mathrm{I}-\mathrm{W})$. From Eigen decomposition of a filtering matrix $\mathrm{W}$, the ith diagonal element $(\lambda \mathrm{i})$ of $\Lambda$ matrix can be represented as $1+\beta(1-\lambda i)$. I+ $\beta(\mathrm{I}-\mathrm{W})$ behaves as sharpening filter on $\mathbf{y}$-Af residuals where $\mathrm{I}-\mathrm{W}$ is high pass filter with $\beta>0$. The fidelity term undergoes different derivatives of the residuals to avoid deblurring problems in real images. Similar analysis is adapted to second term in equation (9) that forms adaptive high pass filters. This results in avoiding unpleasant artifacts due to noise, ringing artifacts, etc and maintains finest details in the restored image with best solution. Its gradient is set to zero by minimizing the cost function at each step

$$
\begin{aligned}
\nabla E(f)= & -2 A^{T}(I+\beta(I-W))(y-A f) \\
& +2 \eta(I-W) f=0
\end{aligned}
$$

which is symmetric and positive definite system of linear equations.

$$
\begin{array}{r}
\left(A^{T}(I+\beta(I-W)) A+\eta(I-W)\right) f \\
=A^{T}(I+\beta(I-W)) y
\end{array}
$$

Further Conjugate Gradient is used and $\mathrm{A}$ and $\mathrm{A}^{\mathrm{T}}$ are interpreted as blurring with their power spectral functions. Experiments are carried out in three outer iterations to get expected deblurred output in many cases.

\section{A. Spectral Analysis}

The minimized cost function in equation (9) is represented as

$$
f^{\prime}=F(A, W) A^{T}(I+\beta(I-W)) y
$$

The significance of equation 12 is that $y$ is filter by $I+\beta(I-W)$, and it is multiplied by transpose of a blurring matrix A through back projection and applied to a symmetric matrix $\mathrm{F}(\mathrm{A}, \mathrm{W})$. The spectral decomposition of symmetric matrix $\mathrm{F}(\mathrm{A}, \mathrm{W})$ is represented as $\Theta^{\prime} \Upsilon \Theta^{\mathrm{T}}$. The spectral filtering analysis of the corresponding deblurring result at each outer step is represented by the columns of a matrix $\Theta$, which serves as orthonormal basis to filter the vector $A^{\mathrm{T}}(\mathrm{I}+\beta(\mathrm{I}-\mathrm{W})) \mathrm{y}$. The basis Eigen vectors in $\Theta$ with their corresponding four largest Eigen values of $\mathrm{F}(\mathrm{A}, \mathrm{W})$ are shown in figure 3. This reveals the data-adaptive character of the filter.

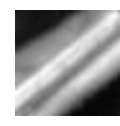

(a)

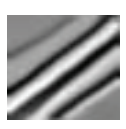

(b)

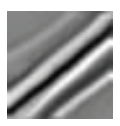

(c)

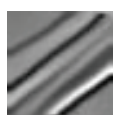

(d)

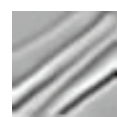

(e)
Figure 2. (a) Original $61 \times 61$ image, (b), (c), (d) and (e) are the Eigenvectors of $F(A, W)$ that corresponds to four largest Eigen values for $\beta$

\section{B. Image Denoising} $=0.7$ and $\eta=0.2$.

With $\mathrm{A}=\mathrm{I}$ in equation 8 , image denoising problem is reduced. Based on SURE-based estimated MSE approach [17], optimal value of regularization parameter $\eta$ is selected. Further improvement can be obtained by iterative approach with some existing kernel based denoising algorithms.

\section{Image Sharpening}

Set $A=I$ and $\eta=0$, then the cost function in equation 8 becomes as

$$
E(f)=(y-f)^{T}(I+\beta(I-W))(y-f)
$$

Simple steepest decent method is used to optimize the above objective function

$$
f^{\prime}=f^{\prime}-1+\mu(I+\beta(I-W))\left(y-f^{\prime}-1\right)
$$

By selecting zero initialization with $\mathrm{f}^{\prime}{ }_{0}=0$ and assigning step size with $\mu=1$, the first iteration is in the form

$$
f^{\prime}=(I+\beta(I-W)) y
$$

Some part of high pass filtered version of the input image is added adaptively with $\beta>0$. W consists of some amount of information of the original image and also about the nature of blurring process. Therefore, data-adaptive sharpening technique was implemented in equation 15 .

\section{IMPLEMENTATION DETAILS}

Initially calculate kernel similarity matrix $\mathrm{M}$, from final estimate of the preceding step, i.e from $f^{\prime M-1}$ for each outer iteration. For all the test images, the regularization parameter values $\eta$ and $\beta$ are kept fixed and these values are chosen based on blurring and noise variance. The parameters $\eta$ and $\beta$ are selected between $[0,0.4]$ and $[0,1]$. Based on the amount of noise and severity of blurring, these values are chosen accordingly. Each step is initialized with suitable estimate from earlier step so that this iterative algorithm converges very quickly. Based on the amount of degradation, the maximum number of outer and inner iterations is set in advance. The iterations will be stopped according to the estimate of Predicted Mean Square Error (PMSE) which is represented as

$$
\operatorname{PS} \widehat{\operatorname{ME}(q}, k)=\frac{\left\|\widehat{A f}-A{f^{\prime}}^{(q)}\right\|^{2}}{n^{2}}
$$

where $A f^{\prime}$ 'is the required deblurred image. The Conjugate Gradient Iterations can be stopped when the following condition is satisfied.

$$
\operatorname{PSM} \widehat{E(q, k}+1)>\operatorname{PSME(q}, k)
$$

\section{RESULTS}

The effectiveness of this algorithm is verified with some number of experiments. Experiments are set up for Gaussian, nonlinear camera motion and out-of-focus blur. To get the final estimate for color image, this algorithm is applied separately to R, G and B channels. These experiments are implemented using MATLAB functions. Structurally Similarity (SSIM) index and PSNR in $\mathrm{dB}$ are used for comparison purposes. A $25 \times 25$ Gaussian blur with standard deviation $\sigma=1.6$ is convolved with a set of color images. A disk function with radius $r=5$ is used to produce Out-of-focus blur and based on [18] motion blur images are generated. These blurred images are added with white Gaussian noise with variance equal to 0.2 and 1 respectively. The experiment is performed by considering i) Patch size of $5 \times 5$, ii) Number of outer iterations as 3, iii) Search neighborhood size as $11 \times 11$ and iv) Number of inner iterations as 30. Table I summarizes $h, \eta, \beta$ parameter values and maximum number of inner CG iterations that are used for Gaussian and out-offocus blur images. 
TABLE I.

DIFFERENT SET OF PARAMETERS IN COLOR IMAGE DEBLURRING METHOD

\begin{tabular}{|l|c|c|c|c|}
\hline \multicolumn{1}{|c|}{ Experiment } & $\eta$ & $\beta$ & $\mathrm{h}$ & $\begin{array}{c}\text { Inner } \\
\mathrm{CG} \\
\text { iteration } \\
\mathrm{s}\end{array}$ \\
\hline Gaussian $(\sigma 2=0.2)$ & 0.003 & 0.23 & 5.3 & 100 \\
\hline Gaussian $(\sigma 2=1)$ & 0.0078 & 0.001 & 7.7 & 100 \\
\hline Motion Blur $(\sigma 2=0.2)$ & 0.005 & 0.41 & 6.2 & 100 \\
\hline Motion Blur $(\sigma 2=1)$ & 0.01 & 0.01 & 6.6 & 80 \\
& & & & \\
\hline
\end{tabular}

This method runs four times faster than implemented on IDDBM3D algorithm on a $2.8 \mathrm{GHz}$ Intel Core $\mathrm{i} 7$ processor. The two-step IDDBM3D algorithm decouples deblurring and denoising process. It depends on suitable estimate from other deblurring algorithm in its grouping phase. But Iterative Graph based method relies on initial denoising and it handles noise amplification issues in much better way. Table II and III summarizes the numerical deblurring results for the images degraded with Gaussian and out-of-focus blur. This method is compared with IDDBM3D algorithm with $\sigma^{2}=0.2$ and $\sigma^{2}=1$. Images of Book-Shelf, Brain Tumor, Human Face, Satellite Image and Scenery are collected. Figure 3 shows the deblurring results for the images degraded with Gaussian blur. The original images are in first row. Gaussian blur images are in second row and third row shows deblurred images. Figure 4 shows Out-of-focus blurred and deblurred images. This algorithm performs better visual quality which can be noticed in smooth face parts of an image. Table IV illustrates the significant results in case of motion deblurring. These are compared with Hyper-Laplacian Method. Figure 5 shows motion blurred and deblurred images. It is observed that Iterative Graph (IG)-based Image Restoration method produces high quality outputs.

TABLE II.

PSNR AND SSIM PERFORMANCE OF AN ITERATIVE GRAPH (IG) BASED METHOD AND IDDBM3D WITH KERNEL SIZE OF 25 X 25 AND WITH STANDARD DEVIATION 1.6 FOR GAUSSIAN BLUR

\begin{tabular}{|c|c|c|c|c|c|c|c|c|}
\hline \multirow{3}{*}{ Blur/Images } & \multicolumn{4}{|c|}{ Gaussian $\left(\sigma^{2}=0.2\right)$} & \multicolumn{4}{|c|}{ Gaussian $\left(\sigma^{2}=1\right)$} \\
\hline & \multicolumn{2}{|c|}{ Iterative Graph-based method } & \multicolumn{2}{|c|}{ IDDBM3D method } & \multicolumn{2}{|c|}{ Iterative Graph-based method } & \multicolumn{2}{|c|}{ IDDBM3D method } \\
\hline & PSNR & SSIM & PSNR & SSIM & PSNR & SSIM & PSNR & SSIM \\
\hline Scenery & 28.99 & 0.9793 & 28.57 & 0.9726 & 28.62 & 0.9683 & 27.89 & 0.9576 \\
\hline Human Face & 33.84 & 0.9756 & 33.18 & 0.9693 & 32.94 & 0.9630 & 32.34 & 0.9522 \\
\hline $\begin{array}{l}\text { Brain Tumor } \\
\text { Image }\end{array}$ & 28.80 & 0.9732 & 27.88 & 0.9677 & 28.34 & 0.9677 & 28.29 & 0.9624 \\
\hline $\begin{array}{l}\text { Satellite } \\
\text { Image }\end{array}$ & 28.36 & 0.9791 & 27.56 & 0.9760 & 27.22 & 0.9675 & 26.24 & 0.9623 \\
\hline Book-Shelf & 27.25 & 0.9827 & 28.25 & 0.9841 & 26.27 & 0.9717 & 27.31 & 0.9787 \\
\hline
\end{tabular}

TABLE III.

PSNR AND SSIM PERFORMANCE OF AN ITERATIVE GRAPH (IG) BASED METHOD AND IDDBM3D WITH DISK FUNCTION OF RADIUS 7 FOR OUT-OF-FOCUS BLUR GENERATED IMAGES

\begin{tabular}{|c|c|c|c|c|c|c|c|c|}
\hline \multirow[t]{3}{*}{ Blur/Images } & \multicolumn{4}{|c|}{ Out-of-focus $\left(\sigma^{2}=0.2\right)$} & \multicolumn{4}{|c|}{ Out-of-focus $\left(\sigma^{2}=1\right)$} \\
\hline & \multicolumn{2}{|c|}{ Iterative Graph-based method } & \multicolumn{2}{|c|}{ IDDBM3D method } & \multicolumn{2}{|c|}{ Iterative Graph-based method } & \multicolumn{2}{|c|}{ IDDBM3D method } \\
\hline & PSNR & SSIM & PSNR & SSIM & PSNR & SSIM & PSNR & SSIM \\
\hline Scenery & 28.64 & 0.9542 & 28.44 & 0.9511 & 27.80 & 0.9369 & 26.90 & 0.9178 \\
\hline Human Face & 33.06 & 0.9489 & 32.28 & 0.9609 & 31.42 & 0.9201 & 30.85 & 0.9058 \\
\hline $\begin{array}{l}\text { Brain Tumor } \\
\text { Image }\end{array}$ & 29.71 & 0.9524 & 29.35 & 0.9514 & 27.13 & 0.9050 & 27.09 & 0.9003 \\
\hline Satellite Image & 28.08 & 0.9609 & 26.92 & 0.9542 & 25.75 & 0.9177 & 24.04 & 0.8974 \\
\hline Book-Shelf & 27.60 & 0.9711 & 28.19 & 0.9709 & 25.63 & 0.9405 & 24.64 & 0.9337 \\
\hline
\end{tabular}


TABLE IV.

PSNR AND SSIM PERFORMANCE OF AN ITERATIVE GRAPH BASED METHOD AND HYPER-LAPLACIAN METHOD FOR CAMERA MOTION BLUR

\begin{tabular}{|c|c|c|c|c|c|c|c|c|}
\hline \multirow{2}{*}{ Blur/Images } & \multicolumn{4}{|c|}{ Motion $\left(\sigma^{2}=0.2\right)$} & \multicolumn{4}{c|}{ Motion $\left(\sigma^{2}=1\right)$} \\
\cline { 2 - 10 } & \multicolumn{2}{|c|}{\begin{tabular}{c} 
Iterative Graph-based method \\
\cline { 2 - 10 }
\end{tabular}} & \multicolumn{2}{|c|}{$\begin{array}{c}\text { Hyper-Laplacian } \\
\text { Method }\end{array}$} & \multicolumn{2}{c|}{$\begin{array}{c}\text { Iterative Graph-based } \\
\text { method }\end{array}$} & \multicolumn{2}{c|}{ Hyper-Laplacian Method } \\
\hline Scenery & 29.47 & 0.9741 & 29.74 & 0.9732 & 28.48 & 0.9521 & 28.43 & 0.9507 \\
\hline Human Face & 33.54 & 0.9636 & 33.53 & 0.9624 & 32.15 & 0.9354 & 31.99 & 0.9351 \\
\hline $\begin{array}{c}\text { Brain Tumor } \\
\text { Image }\end{array}$ & 31.11 & 0.9791 & 31.01 & 0.9766 & 29.75 & 0.9507 & 20.21 & 0.9354 \\
\hline Satellite Image & 29.47 & 0.9743 & 29.37 & 0.9734 & 28.45 & 0.9525 & 28.42 & 0.9507 \\
\hline Book-Shelf & 28.81 & 0.9857 & 29.24 & 0.9839 & 27.31 & 0.9711 & 27.61 & 0.9680 \\
\hline
\end{tabular}

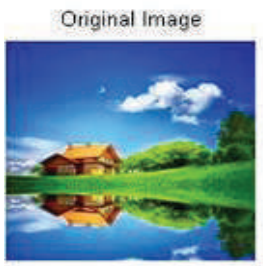

blurred noisy input

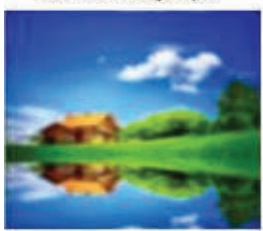

deblurred image

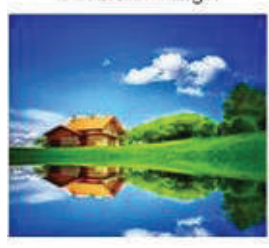

Original Image
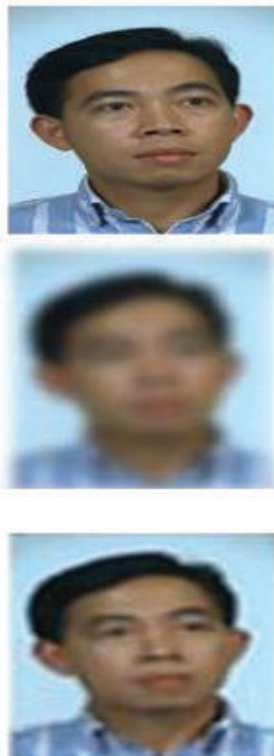

Original Image

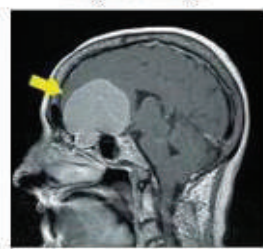

blurred noisy input

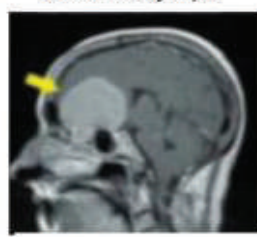

deblurred image

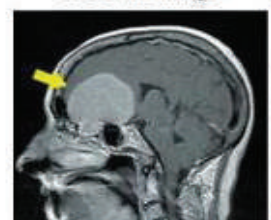

Original Image

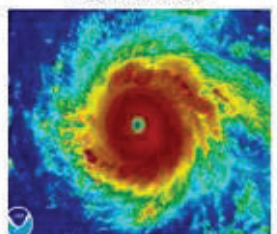

blurred noisy input

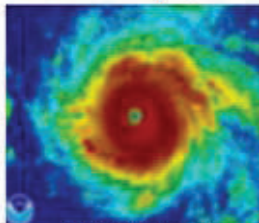

deblurred mage

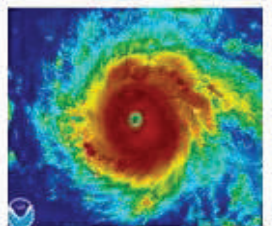

Original Image

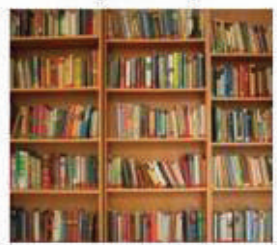

blurred noisy input

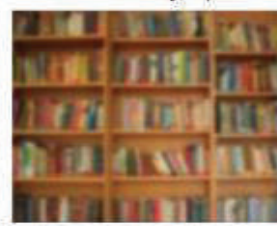

deblurred image

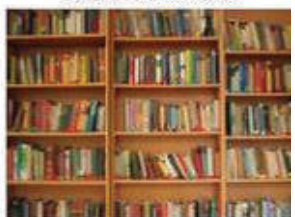

Figure 3. Deblurring example for different Images: First row is Oiginal Image, second row is Gaussian blur Image and third row is deblurred output image

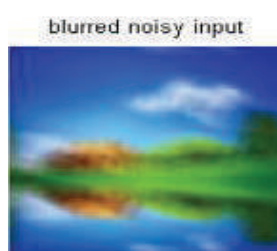

deblurred image

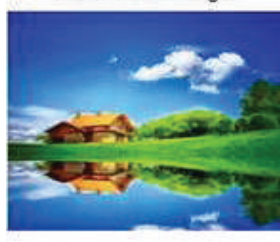

blurred noisy input

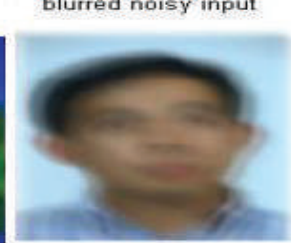

deblurred image

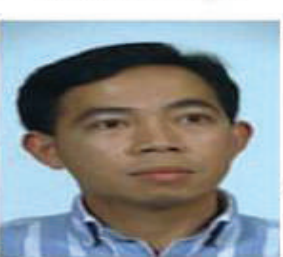

blurred noisy input

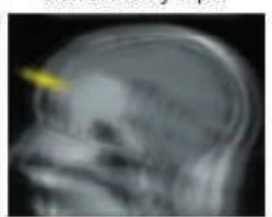

deblurred image

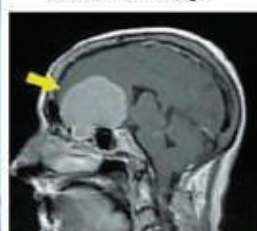

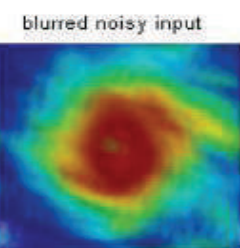

deblurred image

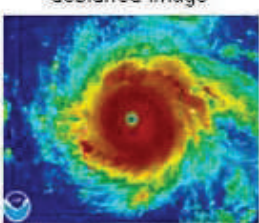

blurred noisy input

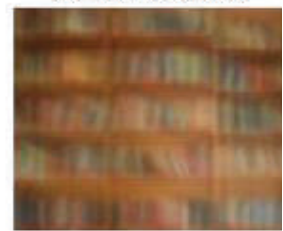

deblurred image

Diाr

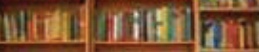

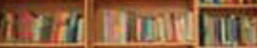

Misis akm

Figure 4. Deblurring examples for Motion Blur: First row are Motion blur Images and second row are deblurred output images 


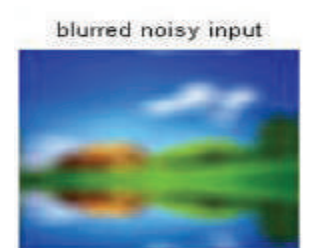

deblurred image

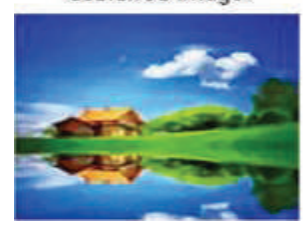

blurred noisy input

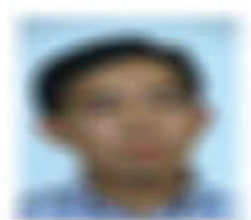

deblurred image

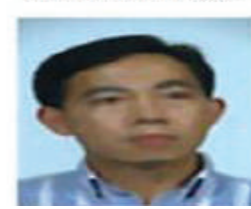

blurred noisy input

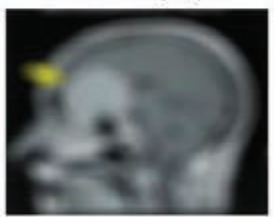

deblurred image

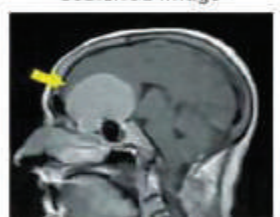

blurred noisy input

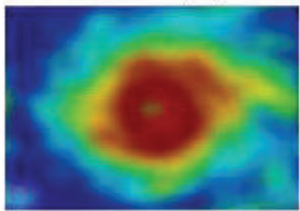

deblurred image

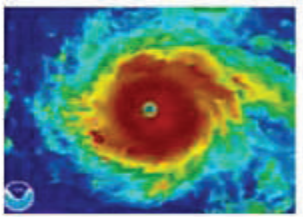

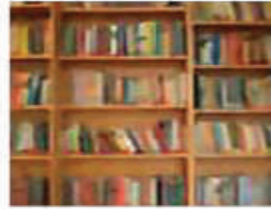

deblurred image

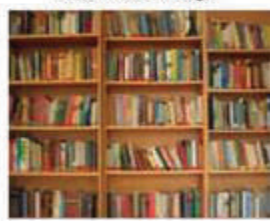

Figure 5. Deblurring examples for Out-of-focus: First row are Out-of-focus blur Images and second row are deblurred output images.

\section{CONClusions}

A wide framework for an Iterative graph based image restoration method has been implemented. An Objective function couples the data and prior terms through Laplacian matrices. The data adaptive approach is improved by graph based filtering approach. This method has been verified for different blurring conditions with some existing state of art algorithms. Based on the appropriate selection of kernel similarity matrix $\mathrm{K}$, many different restoration tasks can be handled. This method can be further extended with blind image deblurring techniques and applied for more complicated non-uniform blurring conditions.

\section{REFERENCES}

[1] G. Chantas, N. P. Galatsanos, R. Molina, and A. K. Katsaggelos, "Variational Bayesian image restoration with a product of spatially weighted total variation image priors," IEEE Trans. Image Process., vol. 19, no. 2, pp. 351-362, Feb. 2010.

[2] W. Dong, L. Zhang, G. Shi, and X. Li, "Nonlocally centralized sparse representation for image restoration," IEEE Trans. Image Process., vol. 22, no. 4, pp. 1620-1630, Apr. 2013.

[3] J. Ni, P. Turaga, V. M. Patel, and R. Chellappa, "Exampledriven manifold priors for image deconvolution," IEEE Trans. Image Process., vol. 20, no. 11, pp. 3086-3096, Nov. 2011.

[4] T. S. Cho, C. L. Zitnick, N. Joshi, S. B. Kang, R. Szeliski, and W. T. Freeman, "Image restoration by matching gradient distributions," IEEE Trans. Pattern Anal. Mach. Intell., vol. 34, no. 4, pp. 683-694, Apr. 2012.

[5] S. Lefkimmiatis, A. Bourquard, and M. Unser, "Hessian-based norm regularization for image restoration with biomedical applications," IEEE Trans. Image Process., vol. 21, no. 3, pp. 983-995, Mar. 2012.

[6] Danielyan, V. Katkovnik, and K. Egiazarian, "BM3D frames and variational image deblurring," IEEE Trans. Image Process., vol. 21, no. 4, pp. 1715-1728, Apr. 2012

[7] A.Levin, R. Fergus, F. Durand, and W. T. Freeman, "Image and depth from a conventional camera with a coded aperture," $A C M$ Trans. Graph., vol. 26, no. 3, Jul. 2007, Art. ID 70.

[8] L. Yuan, J. Sun, L. Quan, and H.-Y. Shum, "Progressive interscale and intra-scale non-blind image deconvolution," $A C M$ Trans. Graph., vol. 27, no. 3, Aug. 2008, Art. ID
[9] Q. Shan, J. Jia, and A. Agarwala, "High-quality motion deblurring from a single image," ACM Trans. Graph., vol. 27, no. 3, pp. 1-10, Aug. 2008, Art. ID 73.

[10] [P. A. Knight and D. Ruiz, "A fast algorithm for matrix balancing," IMA J. Numer. Anal., vol. 33, pp. 1029-1047, 2013.

[11] T. Hofmann, B. Schölkopf, and A. J. Smola, "Kernel methods in machine learning," Ann. Statist., vol. 36, no. 3, pp. 11711220, 2008.

[12] A. Buades, B. Coll, and J. M. Morel, "A review of image denoising algorithms, with a new one," Multiscale Model. Simul., vol. 4, no. 2, pp. 490-530, 2005.

[13] P. Knopp and R. Sinkhorn, "Concerning nonnegative matrices and doubly stochastic matrices," Pacific J. Math., vol. 21, no. 2, pp. 343-348, 1967.

[14] U. Von Luxburg, "A tutorial on spectral clustering", Statistics and computing, vol. 17, no. 4, pp. 395-416, 2007.

[15] P. Milanfar, "A tour of modern image filtering: New insights and methods, both practical and theoretical," IEEE Signal Process. Mag., vol. 30, no. 1, pp. 106-128, Jan. 2013.

[16] S. Bougleux, A. Elmoataz, and M. Melkemi, "Local and nonlocal discrete regularization on weighted graphs for image and mesh processing," Int. J. Comput. Vis., vol. 84, no. 2, pp. 220-236, 2009.

[17] S. Ramani, T. Blu, and M. Unser, "Monte-Carlo SURE: A blackbox optimization of regularization parameters for general denoising algorithms," IEEE Trans. Image Processing, vol. 17, no. 9, pp. 1540-1554, Sep. 2008.

[18] Q. Shan, J. Jia, and A. Agarwala, "High-quality motion deblurring from a single image," ACM Trans. Graph., vol. 27, no. 3, pp. 1-10, Aug. 2008, Art. ID 73. 